\title{
Remanent battery capacity estimation for autonomous ground industrial vehicles
}

\author{
Tom Savu, ${ }^{1, *}$, and Bogdan Alexandru Jugravu ${ }^{1}$ \\ ${ }^{1}$ University POLITEHNICA of Bucharest, Manufacturing Engineering Dept. (TCM), 313 Spl. \\ Independentei, 060042 sector 6, Bucharest, Romania
}

\begin{abstract}
When travelling in an industrial system for completing their assigned tasks, autonomous ground vehicles must estimate the remanent capacity of their batteries and decide if they are able to assume the next task and afterward travel to the charging or replacement station. The amount of energy needed for moving on a certain distance depends on a set of parameters belonging to the vehicle, to the runway and to the vehicle's trajectory. The paper proposes a model for estimating the remaining capacity of the batteries after a certain distance would be covered by a vehicle. Parameters values were obtained by simulation, capacity loss was computed using the proposed model and then a neural network was taught to perform the estimation. The neural network was further used to simulate the situation when a vehicle is estimating the needed capacity before accepting a task to be performed. The results proved that the model and the network, even developed using low data volume and processing time, are able to provide accurate enough estimations and are able to allow future developments.
\end{abstract}

\section{Introduction}

The ground vehicles acting in the actual manufacturing systems, used for moving batches of parts or pieces of equipment between different working places, are in their majority powered by electric batteries. More and more of these vehicles have a certain degree of autonomy, choosing the moving path from one point to another or even choosing or sorting the tasks they are going to accomplish in the following time interval.

One aspect influencing the overall effectiveness of the autonomous ground vehicles $(\mathrm{AGV})$ is represented by the moment when a vehicle must recharge its batteries. Choosing this moment too early leads to a lower value of the vehicle's availability, while postponing the recharge could lead to a total loss of power while performing a task.

Modelling the battery discharge phenomena for an accurate prediction of the battery capacity loss while moving on a certain path could be a complex problem [1] and, even so, the prediction algorithm will need to be fed with data about the vehicle and about the path parameters [2], data which can prove difficult to obtain by measurements.

\footnotetext{
* Corresponding author : savu@ctanm.pub.ro
} 
The paper proposes an estimating procedure which is using a simple model whose parameters are obtained by a neural network, from the data sent by the vehicles moving in the system.

\section{System's behaviour without capacity loss estimation}

Because no real data was available for tests, a previously developed LabVIEW-based simulator [3-4] was used. Initial runs of the simulator were performed without estimating the capacity loss, with the vehicles going to recharge when the battery capacity is less than $20 \%$ at the end of a task.

Figure 1 shows the capacity's variation when a single vehicle is working in the system. Working intervals, during which the capacity is decreasing, are alternating with the recharging intervals.

Figure 2 shows a detailed view of the bottom part of the capacity graph from Figure 1. It is clear that, when checking only of the end of one task if the capacity is lower than $20 \%$, there are tasks with heavier loads and longer distances which are decreasing the capacity as low as $5-6 \%$. Obviously, there exists the risk that the vehicle's batteries will lose their entire capacity during a heavier task, like it can be seen in figure 3 , where the capacities of three vehicles were simulated for a minimum capacity of $10 \%$.

Maintaining the minimum capacity at a higher level, for avoiding the total capacity loss, makes the vehicles to recharge more often so it's decreasing the overall system's effectiveness.

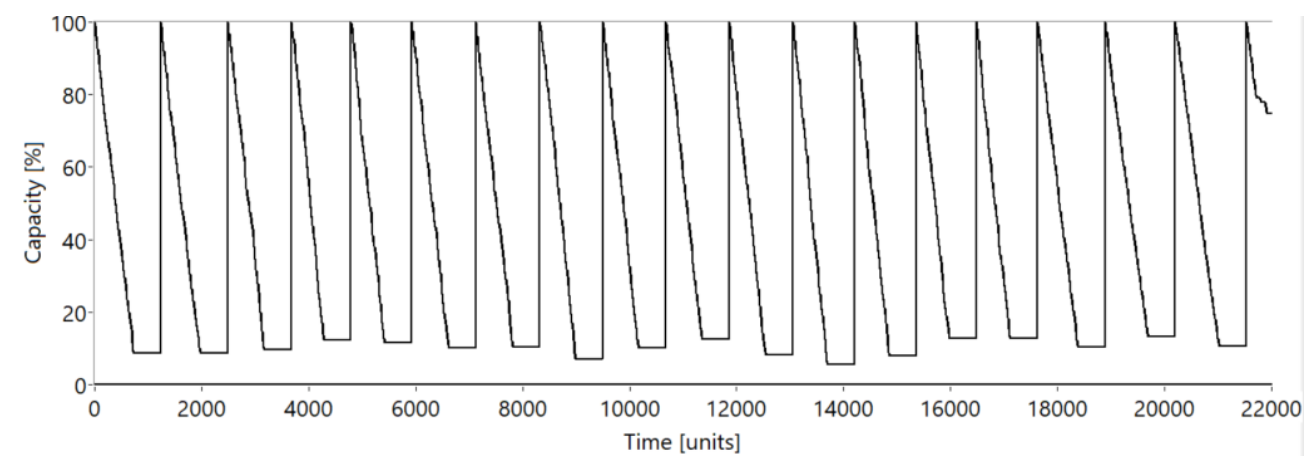

Fig. 1. Capacity variation for a single vehicle.

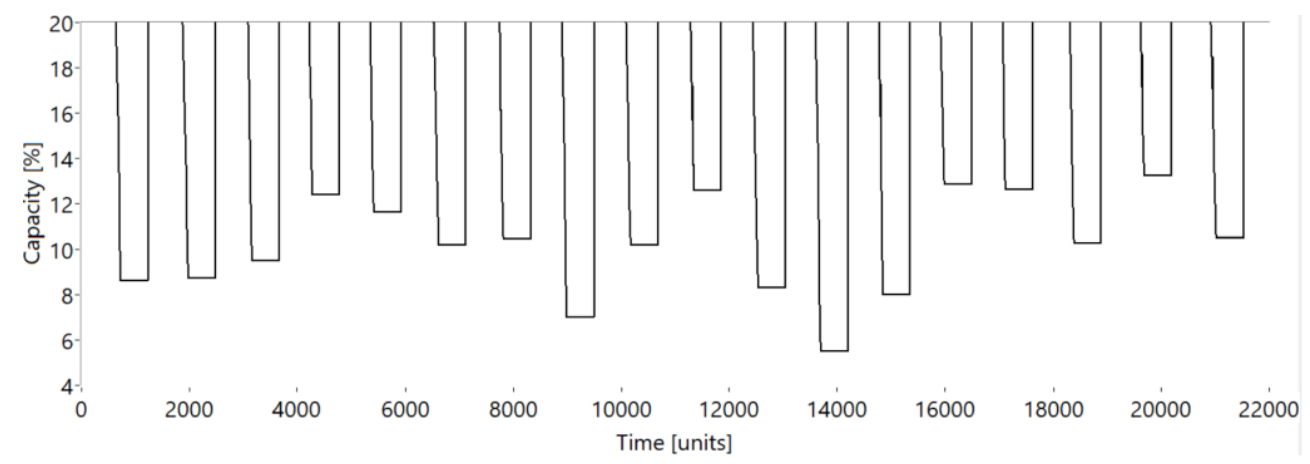

Fig. 2. Detailed view of the capacity graph. 


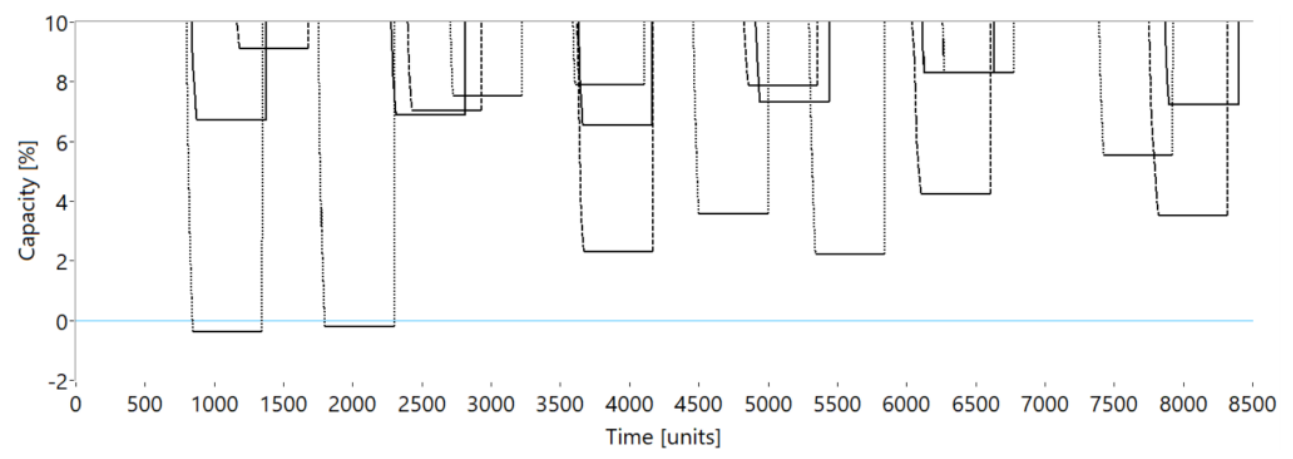

Fig. 3. Example of total capacity loss, in a simulation with three vehicles in the system

\section{Capacity loss simulation}

The proposed model assumes that the capacity loss of a vehicle's battery, for each time unit during the movement on a path segment, can be written as

$$
\delta C=\eta \cdot\left(m \cdot \delta C_{m}+\delta C_{t}\right)
$$

where:

- $\delta C=$ the capacity loss;

- $\eta=$ a coefficient expressing the total influence of path friction, path irregularities and path slope;

- $\mathrm{m}=$ the mass of the transported batch;

- $\delta C_{m}=$ a coefficient expressing the influence of the transported batch's mass;

- $\delta C_{t}=$ the capacity loss due to the transporter's mass and internal energy consumption.

While moving inside the system, every time unit, each vehicle will send data about on which path segment the vehicle is moving, the mass of the transported batch and the $\delta \mathrm{C}$ value of the capacity loss during that time unit.

The vehicle's ID will be also acquired, for accounting the vehicle's mass and its internal energy consuming processes.

The simulator was adapted for generating the data sent by the vehicles and was configured to run with the following parameters:

- $\eta$ coefficient uniformly distributed between 0.7 and 1.3 ;

- the mass of the transported batch uniformly distributed in the $[10 \mathrm{~kg}, 200 \mathrm{~kg}]$ interval;

- $\delta C_{m}$ coefficient uniformly distributed between 0.0005 and 0.0015 ;

- $\delta C_{t}$ uniformly distributed between 0.05 and 0.15 .

A simulation run was performed assuming that three vehicles are working in the system and more than 12500 records were obtained. For running speed reasons, the vehicles were saving the data in CSV-type, comma delimited files.

\section{Neural network teaching}

To prepare the saved data for the neural network processing [5], a specialised procedure is first randomly separating the data in three distinct sets:

- a teaching set, containing $70 \%$ of the available data;

- a validation set, containing $20 \%$ of the available data;

- a testing set, containing the rest of $10 \%$ of the available data. 
The separated data is saved in distinct binary files, each file containing an array of records, each record containing an array with the input values and an array with the expected output (figure 4).

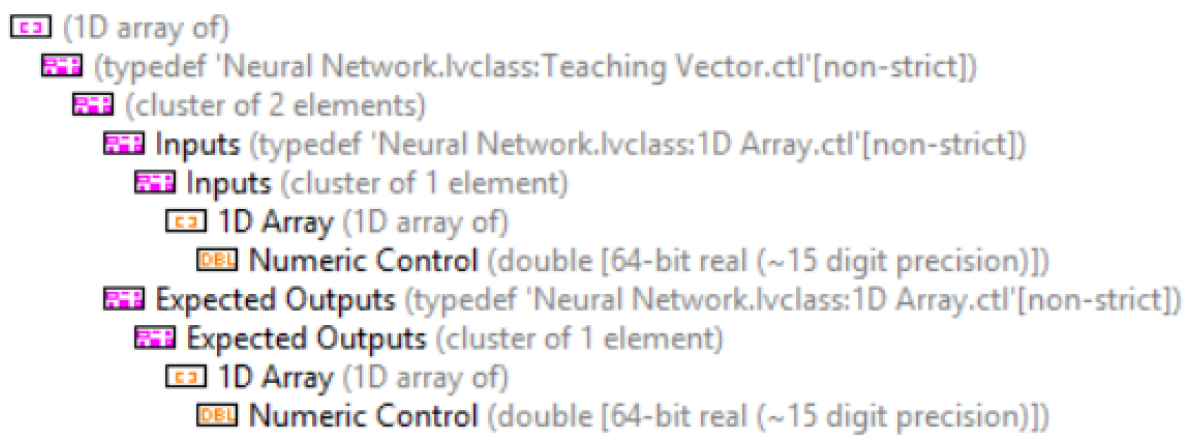

Fig. 4. Binary file structure

The neural network to be trained, developed also in LabVIEW, was one using the resilient backpropagation (RProp) algorithm, with four inputs, one output and three layers, having six neurons on the hidden layer and using an unipolar sigmoidal activation function. The network implements scaling of input and output values to the range of 0.2 to 0.8 , adding bias inputs of 1 to all neurons of all layers.

Network settings were specified for running a maximum of 245,000 epochs, with an error goal of 1 , for a maximum time of 60 seconds, computing 100 vectors per iteration and evaluating the error only over the auto validation set (figure 5).

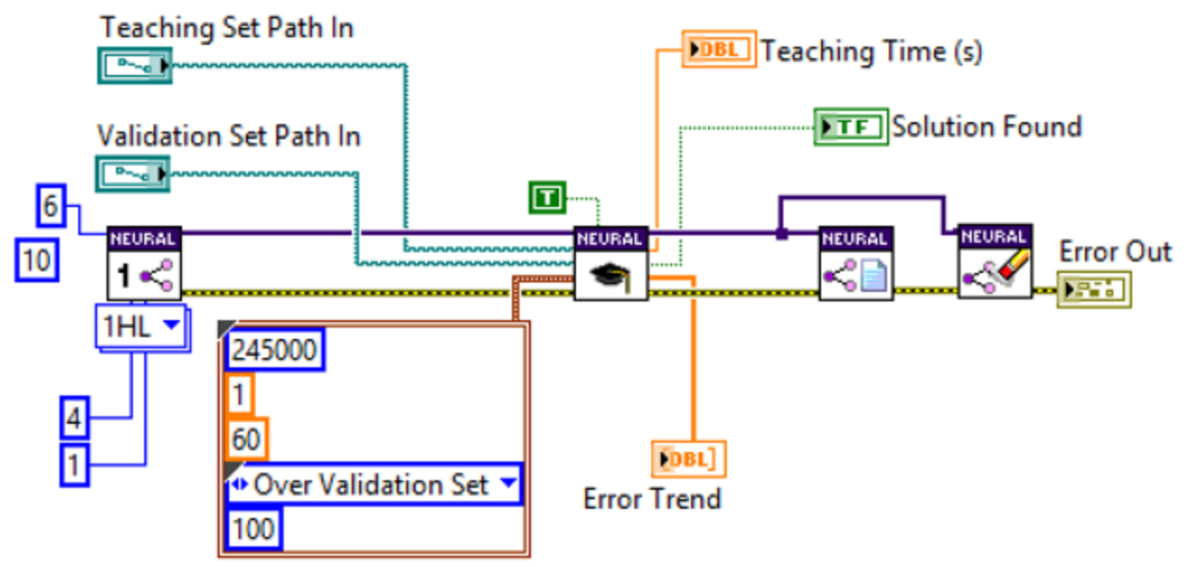

Fig. 5. LabVIEW diagram for teaching the neural network

As seen in figure 6, the network didn't reach the established error goal, since it was difficult to estimate one, but stopped after consuming the maximum running time of 60 seconds. The total error decreased from around 50 at epoch 100 to 46.9 at epoch 656, which indicates that the established goal wasn't realistic, but the network reached a steady state which can be considered satisfactory.

Running the network on the testing data set shows a good enough correspondence between the real data and the computed data (figures 7 and 8), so it was decided that the transporters can use the neural network for estimating the decrease of their capacity during a certain task. 


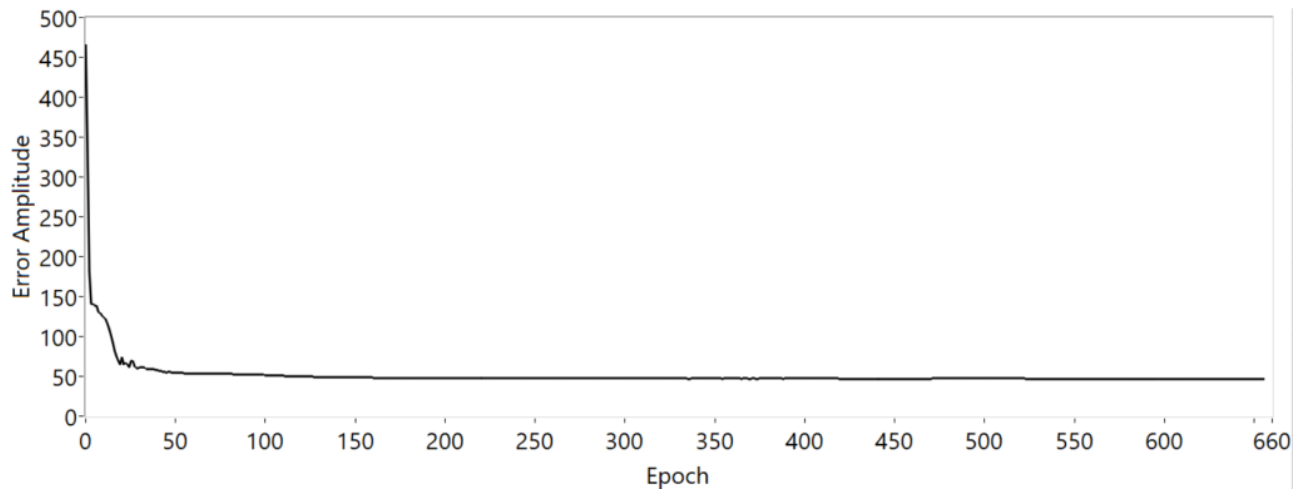

Fig. 6. Neural network error trend during the teaching process

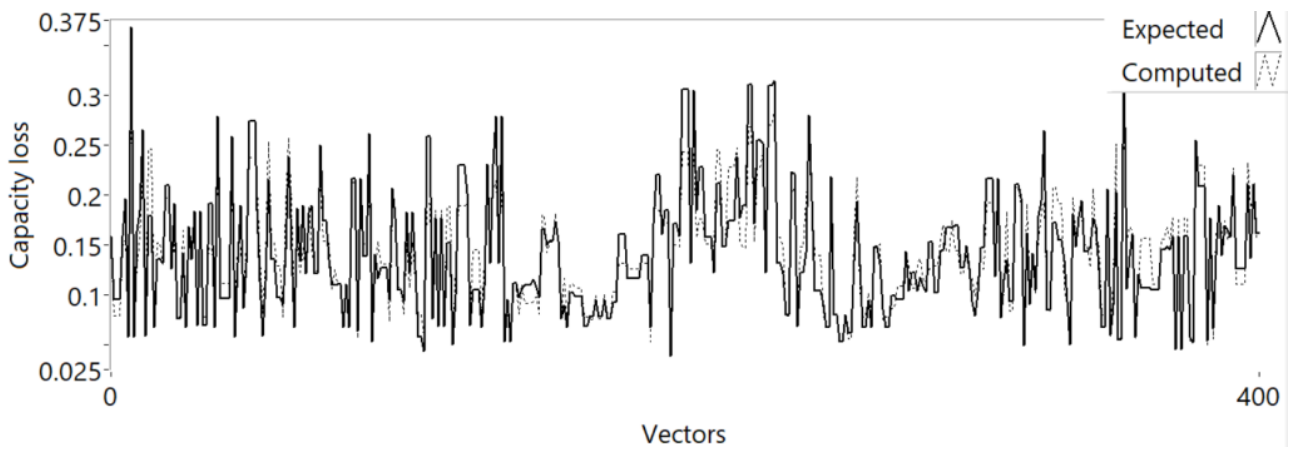

Fig. 7. Expected real data vs. network computed data

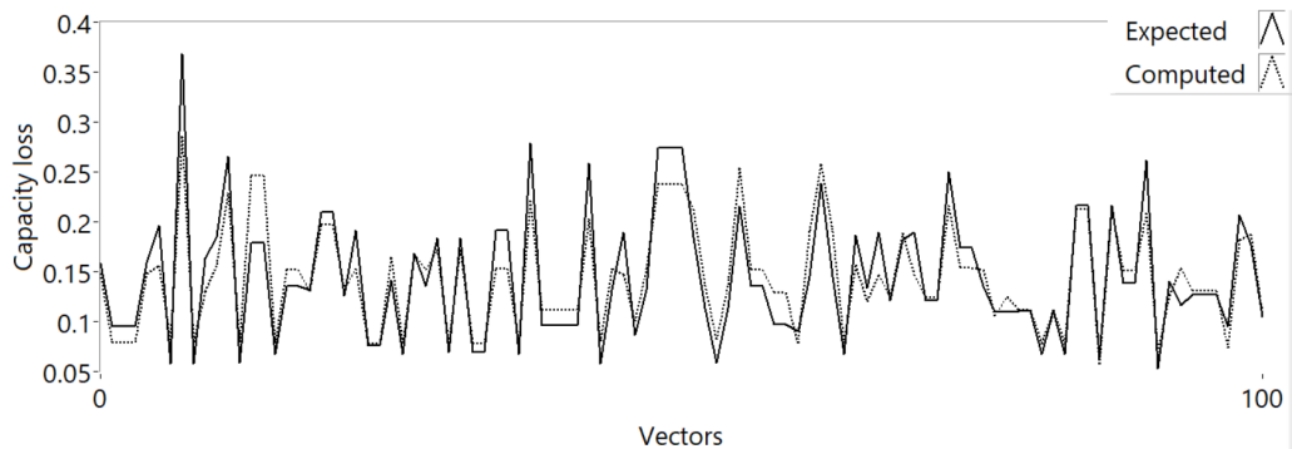

Fig. 8. Detail of the expected real data vs. network computed data graph

\section{Estimating capacity loss by using the neural network}

The simulator was further modified such that, when it must select an order, a vehicle is first estimating the capacity needed to fulfil the order and eventually afterward moving to the recharging station. If the needed capacity is not available, then the vehicle is not accepting the order.

A test simulation for three vehicles in the system shows clearly (figure 9) that now the vehicles are working till lower levels of the capacity, so their usage is more effective, and no total capacity losses appeared. 


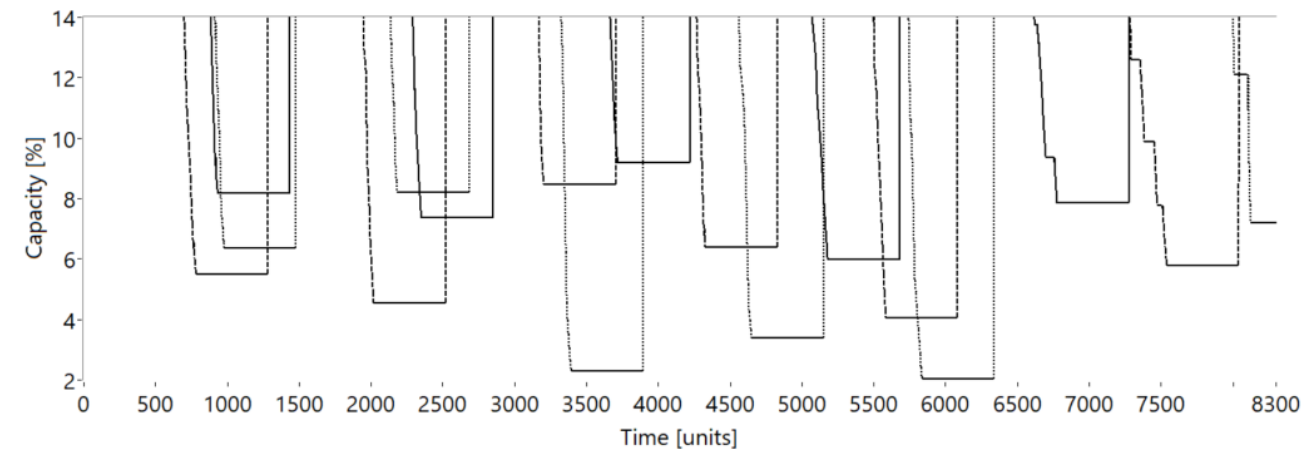

Fig. 9. Test results for three vehicles with capacity estimation

Simulations were further performed for different numbers of vehicles in the system, from one to five, both for the initial situation when no capacity loss estimation is performed and for situation when the loss is estimated. Sets of 30 runs were performed for each combination and mean values of the obtained parameters were computed.

Comparing the total time needed to process all the batches in the system (figure 10), there is only a slight difference for the one vehicle situation, mainly because the total time depends strongly on the batch specific processing time. When more vehicles are present in the system, many of them are just waiting for orders most of the time.

The conclusions change when looking to the mean numbers of rechargings, which are clearly smaller, for all numbers of vehicles, in the situation when the capacity loss is estimated prior to accepting an order (figure 11). This can be explained somehow in terms of energy balance: going to recharge at a lower capacity means that one fully charged battery is more effectively used.

30000

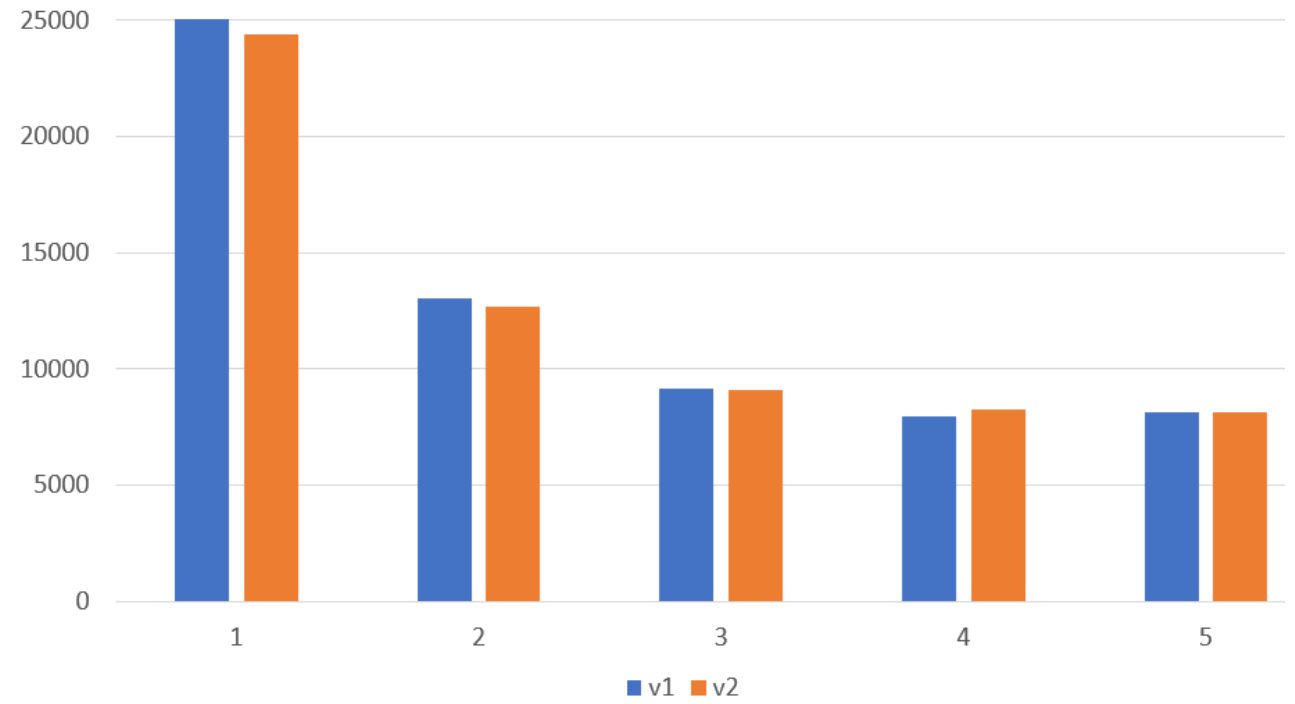

Fig. 10. Total running time vs. number of vehicles 


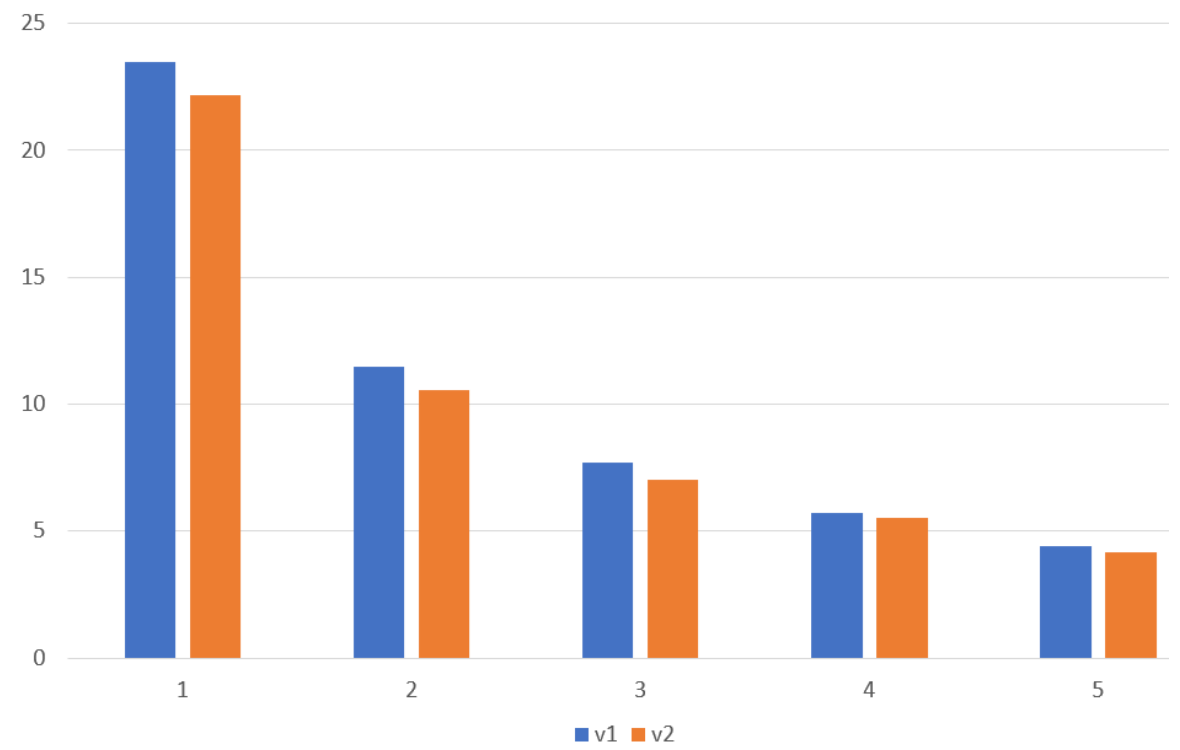

Fig. 11. Mean number of rechargings vs. number of vehicles

\section{Conclusions}

The proposed model for the capacity loss of a vehicle's battery, even if simple and tested with a low volume of simulated data, proved to be one which can be used for reliable estimations.

The structure which was chosen for the neural network allowed, even after a very short teaching session during which a limited amount of data was processed, to obtain a network which provided accurate enough estimations of the capacity loss. The network's goals will be tightened in future developments and its complexity will be increased for obtaining a higher estimation accuracy.

Experiments will be run in the near future for obtaining vehicle data from real situations, thus allowing to choose maybe tighter capacity limits.

The system will be upgraded to compare the estimated capacity loss with that measured by the vehicles, allowing a periodic upgrade of the neural network as the vehicles and path characteristics are changing over time.

\section{References}

1. J. Mange, S. Pace, A. Hundich, P. Sawka, Intl. Conf. on Comp. Sc. and Comp. Intllg. (CSCI), 633-636 (2016)

2. E. Mejri, S. Kelouwani, Y. Dube, O. Trigui, K. Agbossou, IEEE Veh. Power and Prpls Conf. (VPPC) (2017)

3. R. Bitter, T. Mohiuddin, M Nawrocki, LabVIEW: Advanced Programming Techniques, Second Edition (CRC Press - Taylor \& Francis Group, Boca Raton, 2017)

4. T. Savu, B.A. Jugravu, Innov. Mnfg. Eng. \& Energy Intl. Conf. (IManEE) (to be published)

5. M. Hassoun, Fundamentals of Artificial Neural Networks (A Bradford Book, MIT Press, Cambridge, MA, 2013) 